\section{Monitoring a Catalyst at Work}

Jan-Dierk Grunwaldt ${ }^{\star a}$, Stefan Hannemann ${ }^{a}$, Pit Boye ${ }^{b}$, Christian G. Schroer ${ }^{b}$, and Alfons Baikera

${ }^{*}$ Correspondence: PD Dr. J.-D. Grunwaldta,

Tel.: +4144632 30 93, Fax: +41446321163, E-Mail: grunwaldt@chem.ethz.ch, aDepartment of Chemistry and Applied Biosciences, ETH Zurich, CH-8093 Zurich, ${ }^{b}$ Technische Universität Dresden, Institut für Strukturphysik, D-01062 Dresden, Germany

Keywords: Catalysts - Catalytic partial oxidation - EXAFS - Methane $\cdot$ Micro-scale chemical speciation $\cdot$ Structure-activity relationships

Structure-performance relationships gained by studying catalysts at work are considered the key to further development of catalysts. This requires the structural identification of catalysts preferentially under process conditions while measuring the catalytic activity at the same time. In situ X-ray absorption spectroscopy is a well-suited technique for this purpose since it can identify the chemical states of both crystalline and amorphous structures. Up to now studies were performed in an 'integral' way, i.e. averaged over the whole reactor.

Here, we studied the partial oxidation of methane over a $2.5 \mathrm{wt} \%$ $\mathrm{Rh} / \mathrm{Al}_{2} \mathrm{O}_{3}$ catalyst, which is a promising reaction for the production of hydrogen from natural gas. The experimental arrangement and a transmission X-ray image of the catalyst bed are shown in Fig. 1. When the catalyst was heated up in a $\mathrm{CH}_{4} / \mathrm{O}_{2}$ mixture (ratio $2: 1$ ), the reaction to hydrogen and $\mathrm{CO}$ ignited at about $320^{\circ} \mathrm{C}$, and at the same time the Rh particles were reduced. However, a closer look revealed that the $\mathrm{Rh}$ particles at the inlet of the catalyst bed ( $\mathrm{ca} .12$ $\mathrm{mm}$ in length) were oxidized whereas towards the outlet they were in metallic state. This can be extracted from the characteristic spectra for oxidized and reduced Rh (Fig. 1). In a next step we aimed at 2-D mapping of the oxidation state of Rh on a micrometer scale. An Xray camera was installed behind the reactor to record the transmitted intensity with and without the reactor as function of the energy. In this way 160 X-ray absorption images were taken around the $\mathrm{Rh} \mathrm{K}$ edge, four of them being shown in Fig. 2 (top). These X-ray absorption images contain the full absorption spectroscopic information in the XANES region at each point of the reactor. Therefore reconstruction of the spectra and a linear combination fit with spectra for $\mathrm{Rh}^{0}, \mathrm{Rh}^{3+}$ and an uncharacteristic background allows extracting the 2-D distribution of these components (Fig. 2, bottom). Alternatively, also microXAS studies using a beam of a few micrometers in size would provide similar insight into the reactor. A parallelization using dispersive EXAFS or the use of a quick scanning monochromator would, however, be needed for the latter approach. Parallelization in the present study was achieved using the X-ray camera.

The results demonstrate that the structure of a catalyst may vary inside a catalytic reactor. For the first time 2-D mapping of a catalyst bed under catalytic reaction conditions was achieved.

\section{Acknowledgement}

HASYLAB (DESY, Hamburg) is gratefully acknowledged for providing beamtime for this study.

\section{References}

Received: July 20, 2006

J.-D. Grunwaldt, A. Baiker, Catal. Lett. 2005, 5, 99.

J.-D. Grunwaldt, S. Hannemann, C.G. Schroer, A. Baiker, J. Phys. Chem. B 2006, 110,8674 .

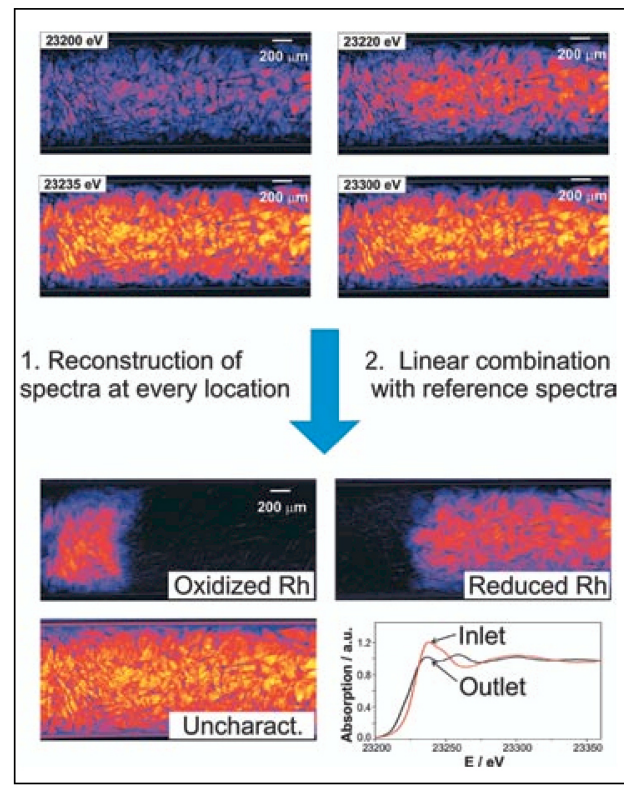

Fig. 2: Selected X-ray transmission images at different energies and the distribution of oxidized and reduced $\mathrm{Rh}$ in the catalyst bed. Uncharacteristic X-ray absorption stems from the absorption of the further elements. (The 160 images used for the reconstruction of the XANES-spectra at each location of the reactor can be downloaded as movie from: $h$ ttp://www. baiker.ethz.ch/people/ Scistaff/Grunwaldt/ ESI.)
Fig. 1: Schematic drawing of the microreactor during partial oxidation of methane with a $2.5 \mathrm{wt} \% \mathrm{Rh} / \mathrm{Al}_{2} \mathrm{O}_{3}$ catalyst (top), an image of the catalyst bed (100-200 $\mu \mathrm{m}$ particles, middle), and characteristic $X$-ray absorption spectra recorded at $362{ }^{\circ} \mathrm{C}$ at the inlet (red, oxidized $\mathrm{Rh}$ ) and the outlet of the reactor (blue, metallic $\mathrm{Rh}$ ). 